\title{
Melting of Discrete Vortices via Quantum Fluctuations
}

\author{
Chaohong Lee, Tristram J. Alexander, and Yuri S. Kivshar \\ Nonlinear Physics Centre and ARC Centre of Excellence for Quantum-Atom Optics, \\ Research School of Physical Sciences and Engineering, Australian National University, Canberra ACT 0200, Australia
}

(Received 11 July 2006; published 1 November 2006)

\begin{abstract}
We consider nonlinear boson states with a nontrivial phase structure in the three-site Bose-Hubbard ring, quantum discrete vortices (or $q$ vortices), and study their "melting" under the action of quantum fluctuations. We calculate the spatial correlations in the ground states to show the superfluid-insulator crossover and analyze the fidelity between the exact and variational ground states to explore the validity of the classical analysis. We examine the phase coherence and the effect of quantum fluctuations on $q$ vortices and reveal that the breakdown of these coherent structures through quantum fluctuations accompanies the superfluid-insulator crossover.
\end{abstract}

DOI: 10.1103/PhysRevLett.97.180408

PACS numbers: 05.30.Jp, 03.75.Lm, 63.20.Pw

Vortices are fundamental objects in physics which appear in different fields including phase singularities in optics [1] and circulating bosons in Bose-Einstein condensates (BECs) [2]. Periodic lattices such as periodic photonic structures for light waves or optical lattices for BECs can modify strongly the wave propagation and may support novel types of vortex states termed discrete vortex solitons $[3,4]$. Such discrete vortices describe spatially localized circular energy flows. They have been studied theoretically and observed experimentally in optically induced photonic lattices as stable self-trapped states of light carrying a nontrivial angular momentum [3]. Similar localized vortex states have been predicted to occur for BECs in optical lattices $[4,5]$.

Since many classical lattice systems supporting discrete vortices originate from quantum models or have a welldefined quantum limit, the fundamental question is: Do these coherent structures survive under the action of quantum fluctuations? In this Letter, we answer this question and reveal the intimate connection between the classical discrete vortex (CDV) and its quantum counterpart, which we call a quantum discrete vortex or $q$ vortex In some sense, these $q$-vortex states can be compared with quantum breathers [6-8], which provide a quantum analog of the self-trapped states with localized energy in discrete classical lattices.

In this Letter, we consider the simplest case of a threesite Bose-Hubbard ring. First, reducing the model to the discrete self-trapping equations [9] with a variational approach, we find CDVs. Next, calculating the spatial correlations in the ground states and the fidelity between the exact and variational ground states, we study the superfluid-insulator crossover and find a valid regime of the classical variational approach. Last, analyzing the phase coherence and quantum fluctuations of the $q$ vortices, we find that the coherent structure "melts" under the action of quantum fluctuations, and this melting process accompanies the superfluid-insulator crossover. This is in sharp contrast to CDVs, whose phase coherence is inde- pendent of the intersite coupling strength and the total number of particles in the vortex.

The basic concepts of the quantum discrete vortices can be captured by the simplest case of a three-site BoseHubbard ring, as shown schematically in the diagrams in Fig. 1. The triple-well potential required for such a state may be readily found as a subset of the familiar Kagomé lattice [10] [see Figs. 1(a) and 1(b)]. For a deep potential, the three-site subsets become decoupled from one another, and each of them obeys the Hamiltonian

$$
H=-T \sum_{\langle l, m\rangle}\left(a_{l}^{+} a_{m}+a_{l} a_{m}^{+}\right)+\frac{U}{2} \sum_{l=1}^{3} n_{l}\left(n_{l}-1\right),
$$

where the operators $a_{l}^{+}$and $a_{l}$ generate and annihilate a particle on the $l$ site, and $T$ and $U$ stand for the tunneling and on-site interaction strengths, respectively. We consider a repulsive interparticle interaction $(U>0)$ assuming the application of our results to BECs.

Our first goal is to connect the classical and quantum pictures for describing the stationary states of this system, and to this end we use the time-dependent variational approach [11]. For the three-site model, we introduce the $\mathrm{SU}(3)$ coherent state

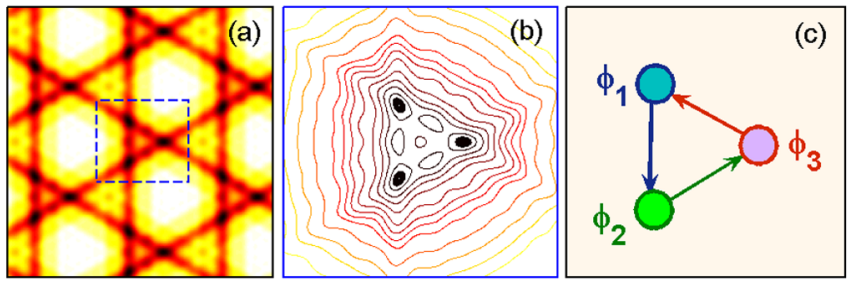

FIG. 1 (color online). Schematic diagrams. (a) Bosons in a strongly trimerized Kagomé lattice [10] can be reduced to threesite Bose-Hubbard rings. (b) Combining a proper twodimensional harmonic potential with the Kagomé lattice, a triple-well potential can be generated; (c) three-site state. 


$$
|\Psi\rangle=\frac{1}{\sqrt{N ! N^{N}}}\left(\sum_{l=1}^{3} \psi_{l} a_{l}^{+}\right)^{N}|v s\rangle,
$$

instead of a conventional product of Glauber's coherent states. Here $N$ is the total number of bosons, $|v s\rangle$ is the vacuum state $\left|n_{1}=0, n_{2}=0, n_{3}=0\right\rangle$, and the complex amplitudes $\psi_{l}$ satisfy the normalization condition $\left|\psi_{1}\right|^{2}+$ $\left|\psi_{2}\right|^{2}+\left|\psi_{3}\right|^{2}=N$. In contrast to the variational approach based on Glauber's coherent states [12], this variational approach conserves the total particle number $N$. Minimizing the corresponding action, we derive the classical Hamiltonian for the complex amplitudes $\psi_{l}$

$$
H_{c}=-T \sum_{\langle l, m\rangle}\left(\psi_{l}^{*} \psi_{m}+\psi_{l} \psi_{m}^{*}\right)+\frac{U_{N}}{2} \sum_{l=1}^{3}\left|\psi_{l}\right|^{4},
$$

where $U_{N} \equiv(N-1) U / N$. The extra factor $(N-1) / N$ does not appear in the Hamiltonian obtained from the variational approach using Glauber's coherent states. The complex amplitudes $\psi_{l}$ obey the three-site discrete selftrapping equations $[9,13,14]$ :

$$
i \frac{d \psi_{l}}{d t}=-T \sum_{m \neq l} \psi_{m}+U_{N}\left|\psi_{l}\right|^{2} \psi_{l},
$$

with $\{l, m\}=1,2,3$. We are interested in the stationary solutions and therefore make the substitution $\psi_{l}=$ $A_{l} \exp \left[-i\left(\mu t-\phi_{l}\right)\right]$ with chemical potential $\mu$, nonnegative real amplitudes $A_{l}$, and phases $\phi_{l}$. The system of equations (4) has a well-known degenerate set of solutions with equal amplitudes [15]. The solutions with $\phi_{1}=$ $\phi_{2}=\phi_{3}, \quad A_{1}=A_{2}=A_{3}=\sqrt{N / 3}, \quad$ and $\quad \mu=(N-$ 1) $U / 3-2 T$ describe the ground state of the system. The symmetric vortex states have $A_{1}=A_{2}=A_{3}=$ $\sqrt{N / 3}, \phi_{2}-\phi_{1}=\phi_{3}-\phi_{2}=2 l \pi / 3$ (where $l$ are nonzero integers), and $\mu=(N-1) U / 3-2 T \cos (2 l \pi / 3)$. These three-site CDVs exist for arbitrary values of $N, U$, and $T$. There exist also asymmetric solutions which have only two equal amplitudes. These states are the excited states of the system and ultimately connect with localization maintained by self-trapping [13].

We now return to the fully quantum description for the Hamiltonian (1), in which the behavior depends on the ratio $U / T$ and $N$. The most prominent effect in infinite quantum systems is the superfluid-insulator transition. In our finite-sized system, the ground state is indeed superfluid in the strong tunneling limit $U / T \ll 1$, as expected from the infinite case. In the weak-tunneling limit $U / T \gg$ 1 , the ground state is insulating for commensurate cases $(N=3 k$, with a positive integer $k$ ) but has a small superfluid fraction accompanying an insulating core for incommensurate cases $(N \neq 3 k)$. However, instead of a sharp phase transition observed for infinite systems, these two limits are connected by a crossover regime. That is to say, the superfluid-insulator crossover in this finite-site system with commensurate fillings takes the place of the superfluid-insulator transition predicted and observed in the infinite systems [16], and, hence, there is no welldefined critical value of the ratio $U / T$ between the superfluid and insulating phases. Using exact numerical diagonalization, we calculate the spatial correlations $\left|\left\langle a_{i}^{+} a_{i+1}\right\rangle\right| / n_{\mathrm{av}}$, where $n_{\mathrm{av}}=N / 3$ is the average number of particles per site; see Fig. 2. These results show that in the limit of weak tunneling the superfluid fraction depends strongly on $N$ and the spatial correlations decrease with $U / T$ such that the system approaches its classical counterpart analyzed above.

As the first step to compare the classical results found using the variational approach with those obtained using exact diagonalization, we compare the ground-state energies. Denoting $E_{\mathrm{va}}^{G}$ the ground-state energy obtained from the variational approach and $E_{\mathrm{ex}}^{G}$ that obtained by exact diagonalization, we show that the fractional difference of the ground-state energies $\left(1-E_{\mathrm{va}}^{G} / E_{\mathrm{ex}}^{G}\right)$ increases with the ratio $U / T$ close to the linear limit $U=0$. In the limit of strong tunneling, i.e., for $U / T \ll 1$, the results show excellent agreement between the two approaches. This is also confirmed by the fidelity between the exact and variational ground states $F=\left.\left.\right|_{\mathrm{va}}\langle\mathrm{GS} \mid \mathrm{GS}\rangle_{\mathrm{ex}}\right|^{2}$, which decreases with $U / T$ for arbitrary $N$; see Fig. 3. With $U / T<0.1995$, for $N$ from 1 to $18,\left(1-E_{\mathrm{va}}^{G} / E_{\mathrm{ex}}^{G}\right)<8 \times 10^{-3}$ and $F>0.9790$. The fidelity decrease with $U / T$ indicates that the breakdown of the variational approximation accompanies the occurrence of the superfluid-insulator crossover.

Now we can construct the quantum vortex states. The fully quantum counterparts for symmetric CDVs can be presented as $\mathrm{SU}(3)$ coherent states $|\Psi\rangle$ with complex amplitudes $\psi_{k}=\sqrt{N / 3} \times \exp [i(\varphi+2(k-1) L \pi / 3)]$, with $k$ $(=1,2,3)$ and $L$ denoting the site index and the vortex charge, respectively. Such $q$ vortices,

$$
|\mathrm{QDV}\rangle=\sum_{n_{2}=0}^{N} \sum_{n_{3}=0}^{N-n_{2}} C\left(n_{2}, n_{3}\right)\left|n_{1}, n_{2}, n_{3}\right\rangle,
$$

can be prepared in the limit of strong tunneling by im-
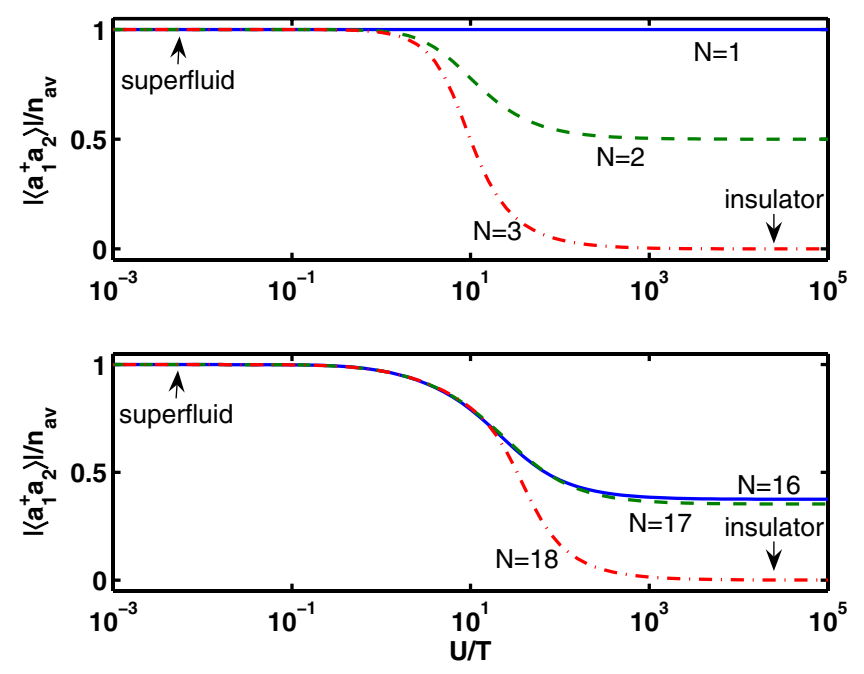

FIG. 2 (color online). Spatial correlations vs $U / T$. 


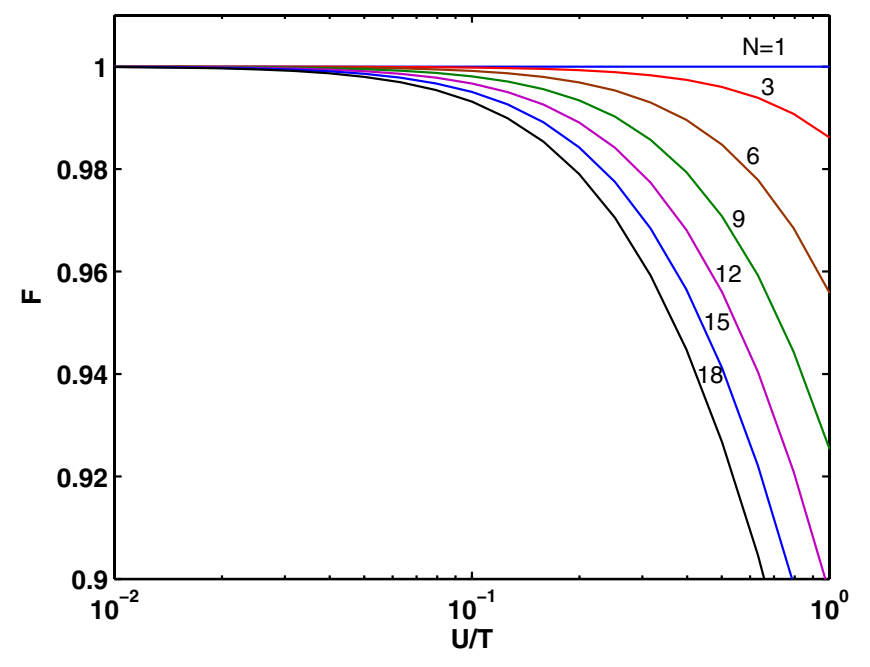

FIG. 3 (color online). Fidelities between the variational and exact ground states vs $U / T$ for different $N$.

printing the specific phase structure $(\varphi, \varphi+2 L \pi / 3$, $\varphi+4 L \pi / 3)$ onto the ground states $|\mathrm{GS}\rangle=$ $\sum \sum G\left(n_{2}, n_{3}\right)\left|n_{1}, n_{2}, n_{3}\right\rangle$, and the coefficients satisfy

$$
C\left(n_{2}, n_{3}\right)=G\left(n_{2}, n_{3}\right) \exp \left\{i\left[N \varphi+\frac{2}{3} n_{2} L \pi+\frac{4}{3} n_{3} L \pi\right]\right\},
$$

where $N=n_{1}+n_{2}+n_{3}$. Fast phase imprinting of an atomic cloud with a required phase structure via atom-laser interaction was also suggested for entanglement preparation [17], as well as atomic dark-soliton generation and measurement [18]. The asymmetric $q$ vortices can be obtained by applying similar phase imprinting procedures to excited states whose classical limit (strong tunneling limit) correspond to asymmetric solutions. Below, we consider only symmetric vortices.

In the linear limit, the $q$ vortices with $L=3 k$ ( $k$ are integers) are ground states of the system, while those with $L=3 k \pm 1$ correspond to the excited states with higher energies. Beyond the linear limit, the quantum counterparts for CDVs can be obtained by quantum adiabatic evolution. For our small-size systems, we simulate the time evolution in the complete Hilbert space with the Runge-Kutta integration scheme [19]. We change the parameters adiabatically such that the populated state is always very close to an eigenstate. To determine whether a given state or even the quantum vortex itself is a well-defined eigenstate, we project it onto a complete Hilbert basis of an ensemble of the orthogonal eigenstates. In the linear limit, we find that all nonzero-probability components of the vortex have identical eigenvalues, and, therefore, we may conclude that the $q$ vortex is an eigenstate. Adiabatically varying $U / T$ to the strongly nonlinear limit, these $q$ vortices evolve into different final states dependent on the charges $L$. For $L=3 k$, the final states are single-peaked states in the distribution of the probability amplitudes $\left|C\left(n_{1}, n_{2}\right)\right|^{2}$ (the ground state). However, for $L=3 k \pm 1$, the quantum vortex (5) appears to be a well-defined eigenstate with a desired probability $P_{\mathrm{es}}$ if $U / T$ is less than a certain value.
For $N=6, P_{\text {es }}>0.9850$ when $U / T<0.1259$. As the nonlinearity is adiabatically increased, the $q$ vortex breaks down, ending up in the limit of strong nonlinearity as a triple-peaked state in the probability distribution. The appearance of the single-peaked and triple-peaked states is independent of the total atom number; it indicates the loss of the circular current of particles and effective melting of the discrete vortex structure. In Fig. 4, we show the quantum adiabatic evolution of the $q$ vortices with $N=6$.

To explore in detail how the classical discrete vortices "melt," we calculate quantum fluctuations of their quantum counterparts, i.e., the $q$ vortices. Using the quantum phase concept [20], we introduce the cosine and sine functions of the quantum phase $\phi_{j}$ for the $j$ th site as $\cos \phi_{j}=\left(K_{j} / 2\right)\left(a_{j}^{+}+a_{j}\right)$ and $\sin \phi_{j}=\left(i K_{j} / 2\right)\left(a_{j}^{+}-a_{j}\right)$, with the constant $K_{j}$ determined by the particle number. Thus, the cosine and sine functions for the two-body phase difference $\phi=\phi_{2}-\phi_{1}$ can be defined as

$$
\begin{gathered}
\cos \phi=K\left(a_{2}^{+} a_{1}+a_{2} a_{1}^{+}\right), \\
\sin \phi=i K\left(a_{2}^{+} a_{1}-a_{2} a_{1}^{+}\right),
\end{gathered}
$$

where the constant $K=K_{1} K_{2}$. Using the conservation character of the square summary $\left\langle\sin ^{2} \phi+\cos ^{2} \phi\right\rangle=1$, it is easy to obtain $K_{1} K_{2}=1 / \sqrt{2\left\langle 2 n_{1} n_{2}+n_{1}+n_{2}\right\rangle}$.

The expectation value and the variance of $\cos \phi$ are

$$
\langle\cos \phi\rangle=\frac{\left\langle a_{2}^{+} a_{1}+a_{2} a_{1}^{+}\right\rangle}{\sqrt{2\left\langle 2 n_{1} n_{2}+n_{1}+n_{2}\right\rangle}}
$$

and

$$
\Delta(\cos \phi)=\left\langle\cos ^{2} \phi\right\rangle-\langle\cos \phi\rangle^{2},
$$

respectively, where the expectation value for $\cos ^{2} \phi$ is

$$
\left\langle\cos ^{2} \phi\right\rangle=\frac{1}{2}+\frac{\left\langle\left(a_{2}^{+} a_{1}\right)^{2}\right\rangle+\left\langle\left(a_{2} a_{1}^{+}\right)^{2}\right\rangle}{2\left\langle 2 n_{1} n_{2}+n_{1}+n_{2}\right\rangle} .
$$

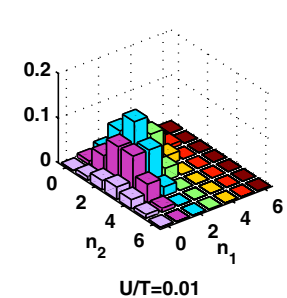

(a) ground state
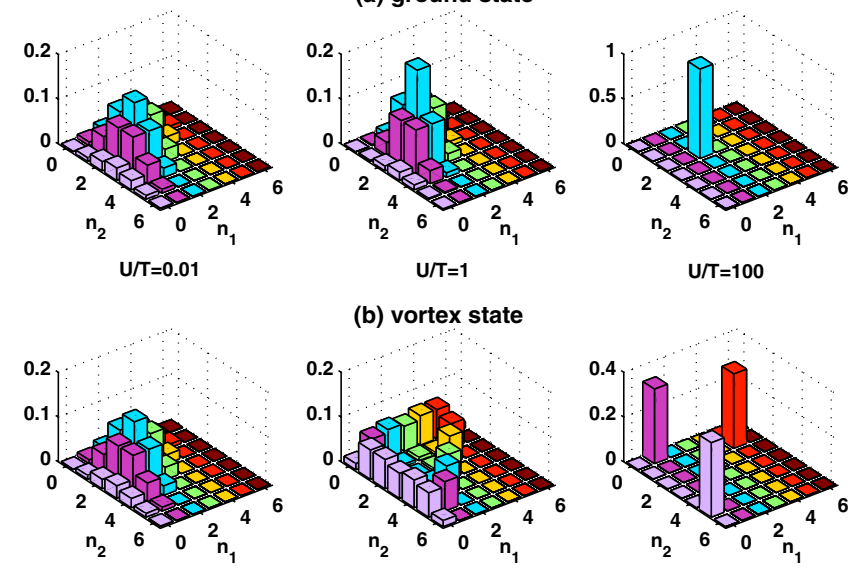

(b) vortex state
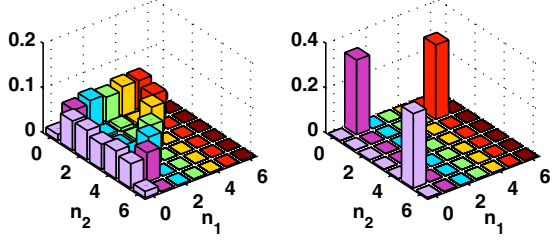

FIG. 4 (color online). Quantum adiabatic evolution of $q$ vortices with $N=6$ from the linear to the nonlinear limit. (a) and (b) correspond to the cases of $L=3 k$ and $L=3 k \pm 1$, respectively. 

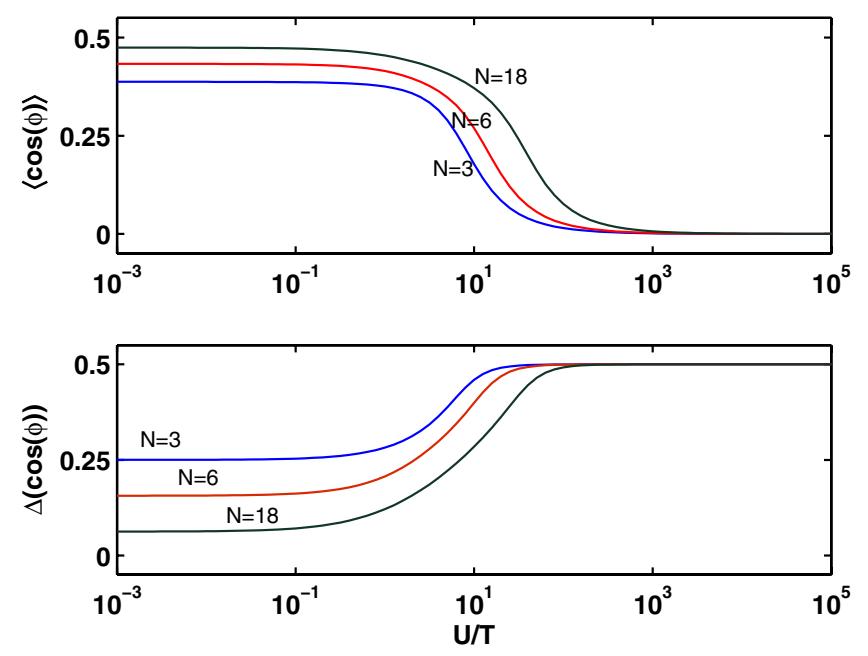

FIG. 5 (color online). Quantum fluctuations for the vortex states vs the ratio $U / T$ for different values of $N$.

If the matter waves located at two neighboring sites have a well-defined phase difference $\phi$, the variance of $\cos \phi$ vanishes. Otherwise (e.g., when the values of $\phi$ are random), the expectation value for $\cos \phi$ and the corresponding variance will approach zero and $1 / 2$, respectively. The random distribution of the phase difference means melting of the coherence between the matter-wave clouds localized at the neighboring sites.

By adiabatically increasing $U / T$ for the $q$ vortices (5) with the charge $L=1$, we calculate numerically the expectation values $\langle\cos \phi\rangle$ and variances $\Delta(\cos \phi)$ between sites 1 and 2 [where $\cos \phi=\cos \left(\phi_{2}-\phi_{1}\right)$ ] for different $U / T$ starting from the linear limit. For the commensurate cases, the expectation value $\langle\cos \phi\rangle$ grows with $N$ and decreases with $U / T$, but the variance $\Delta(\cos \phi)$ decreases with $N$ and increases with $U / T$, as shown in Fig. 5. These results mean that quantum fluctuations decrease with $N$ but increase with $U / T$. In the limit of strong tunneling, i.e., for $U / T \ll 1$, the expectation values $\langle\cos \phi\rangle \rightarrow \cos (2 \pi / 3)$ and variances $\Delta(\cos \phi) \rightarrow 0$ for $N \rightarrow \infty$. The limit of strong tunneling with large total particle numbers corresponds to the classical limit with well-defined phases. The opposite occurs in the limit of weak tunneling $(U / T \gg 1)$ where the expectation values $\langle\cos \phi\rangle \rightarrow 0$ and variances $\Delta(\cos \phi) \rightarrow 1 / 2$ for arbitrary $N$. This means that the distribution of the phase differences becomes random, and the classical discrete vortices melt completely under the action of strong quantum fluctuations. Varying $U / T \ll 1$ to $U / T \gg 1$, we recover the crossover regime that connects these two limits.

We suggest that these effects can be studied experimentally by loading an ${ }^{87} \mathrm{Rb}$ condensate into the triple-well potential formed by a superposition of a harmonic potential (with frequency $\omega \sim 300 \times 2 \pi \mathrm{Hz}$ ) and a Kagomé lattice [10]; see Fig. 1. The Kagomé lattice can be formed by laser beams with wavelengths of $1064 \mathrm{~nm}$. The ratio $T / U$ can then be adjusted by varying the laser intensity about from 1.5 to $15 \mathrm{~W} / \mathrm{cm}^{2}$ to observe the crossover regime and the melting of the vortex phase.

In conclusion, we have studied a quantum analog of discrete vortices as the states of interacting bosons with a nontrivial phase structure. We have introduced the $q$ vortices for the simplest case of a three-site Bose-Hubbard ring and analyzed the effect of quantum fluctuations on these states. We have found that the melting of discrete vortices via quantum fluctuations accompanies the crossover from superfluid to Mott insulator. We believe our findings may initiate experimental efforts to observe quantum discrete vortices in Bose-Einstein condensates in optical lattices.

We thank E. A. Ostrovskaya, A. A. Sukhorukov, L. Santos, Y.-Z. Zhang, P. Drummond, and J. Corney for discussions, R. Gati for estimation of experimental parameters, and the Australian Research Council for support.

[1] M. S. Soskin and M. V. Vasnetsov, in Progress in Optics, edited by E. Wolf (North-Holland, Amsterdam, 2001), Vol. 42, p. 219.

[2] K. W. Madison et al., Phys. Rev. Lett. 84, 806 (2000); C. Raman et al., Phys. Rev. Lett. 87, 210402 (2001).

[3] D. Neshev et al., Phys. Rev. Lett. 92, 123903 (2004); J. W. Fleischer et al., Phys. Rev. Lett. 92, 123904 (2004).

[4] E. A. Ostrovskaya and Yu. S. Kivshar, Phys. Rev. Lett. 93, 160405 (2004).

[5] T. J. Alexander et al., Phys. Rev. A 72, 043603 (2005).

[6] W.Z. Wang et al., Phys. Rev. Lett. 76, 3598 (1996).

[7] V. Fleurov, Chaos 13, 676 (2003).

[8] J. Dorignac et al., Phys. Rev. Lett. 93, 025504 (2004).

[9] J. C. Eilbeck et al., Physica (Amsterdam) 16D, 318 (1985).

[10] L. Santos et al., Phys. Rev. Lett. 93, 030601 (2004); B. Damski et al., Phys. Rev. A 72, 053612 (2005).

[11] P. Buonsante et al., Phys. Rev. A 72, 043620 (2005).

[12] L. Amico and V. Penna, Phys. Rev. Lett. 80, 2189 (1998).

[13] C. Schmidt-Hattenberger et al., J. Opt. Soc. Am. B 10, 1592 (1993).

[14] R. Franzosi and V. Penna, Phys. Rev. E 67, 046227 (2003).

[15] L. Castti and V. Penna, J. Low Temp. Phys. 126, 455 (2002).

[16] M.P. A. Fisher et al., Phys. Rev. B 40, 546 (1989); D. Jaksch et al., Phys. Rev. Lett. 81, 3108 (1998); M. Greiner et al., Nature (London) 415, 39 (2002).

[17] K. W. Mahmud, M.A. Leung, and W.P. Reinhardt, cond-mat/0403002.

[18] S. Burger et al., Phys. Rev. Lett. 83, 5198 (1999); J. Denschlag et al., Science 287, 97 (2000).

[19] C. Leforestier et al., J. Comput. Phys. 94, 59 (1991); S. R. Manmana et al., in Lectures on the Physics of Highly Correlated Electron Systems IX: Ninth Training Course in the Physics of Correlated Electron Systems and High-T Superconductors, edited by A. Avella and F. Mancini, AIP Conf. Proc. No. 789 (AIP, New York, 2005), p. 269.

[20] S. M. Barnett and D. T. Pegg, J. Phys. A 19, 3849 (1986). 